\title{
FOXO1 and ANGPT2 relative gene expression in non-ST-segment elevation myocardial infarction among patients with or without type 2 diabetes
}

\author{
Tomasz Skowerski ${ }^{1,2}$, Katarzyna Nabrdalik ${ }^{2}$, Hanna Kwiendacz ${ }^{2}$, Maciej Pajak ${ }^{3}$, Andrzej Ochała ${ }^{4}$, \\ Katarzyna Mizia-Stec ${ }^{5}$, Zbigniew Gąsior ${ }^{1}$, Janusz Gumprecht ${ }^{2}$ \\ ${ }^{1}$ Department of Cardiology, School of Health Sciences, Medical University of Silesia, Katowice, Poland \\ ${ }^{2}$ Department of Internal Medicine, Diabetology and Nephrology, Faculty of Medical Sciences in Zabrze, Medical University of Silesia \\ in Katowice, Poland \\ ${ }^{3}$ Roslin Institute, Midlothian, Edinburgh, United Kingdom of Great Britain and Northern Ireland \\ ${ }^{4}$ Division of Cardiology and Structural Heart Diseases Medical University of Silesia, Katowice, Poland \\ ${ }^{5}$ First Department of Cardiology, Faculty of Medical Sciences, Medical University of Silesia, Katowice, Poland
}

Adv Interv Cardiol 2021; 17, 2 (64): 187-192

DOI: https://doi.org/10.5114/aic.2021.107498

\section{A bstract}

Introduction: It is well known that chronic hyperglycemia or chronic inflammation leads to both FOXO1 and Ang-2 gene (ANGPT2) expression induction in endothelial cells. ANGPT2 and FOXO1 relative gene expression in peripheral blood cells in diabetes and myocardial ischemia were not researched extensively.

Aim: Our objective was to evaluate ANGPT2 and FOXO1 gene expression in peripheral blood cells in patients with non-ST elevation myocardial infarction (NSTEMI), both with and without type 2 diabetes mellitus (T2DM), and compare them to the results obtained from T2DM and control subjects.

Material and methods: This was a multi-center, prospective study of 138 NSTEMI patients with/without T2DM, T2DM and a control group. FOXO1, ANGPT2, TBP (TATA box binding protein - as a reference gene) gene expression levels in peripheral blood cells were measured in each patient. Electrocardiography and echocardiography with assessment of ejection fraction (EF) were performed. Patients with NSTEMI underwent urgent $(<24 \mathrm{~h})$ coronarography and the SYNTAX score and GRACE 2.0 score were calculated.

Results: The ANGPT2 gene relative expression in buffy coat in the analyzed samples was very low and detectable only in 11 patients from all groups (8.66\% of all patients). The level of FOXO1 gene relative expression was significantly higher in patients with NSTEMI (median relative expression $=1.39)$ than in non-NSTEMI patients (median $=1.09)(W=1578, p<0.05)$ regardless of the presence of T2DM. The FOXO1 gene relative expression was not correlated with GRACE 2.0 score or SYNTAX score of NSTEMI patients. We did not observe any significant change in FOXO1 gene expression after successful angioplasty.

Conclusions: On the basis of our results we can conclude that analyzing the ANGPT2 gene relative expression in peripheral blood cells has no role in assessment of CAD complexity among patients with and without T2DM. FOXO1 gene relative expression in blood peripheral cells is elevated in patients with NSTEMI regardless of the presence of T2DM. FOXO1 expression does not decrease after successful percutaneous coronary intervention and is not correlated with the severity of CAD in patients with NSTEMI.

Key words: angiopoietin-2, diabetes, foxo-1, non-ST elevation myocardial infarction.

\section{Introduction}

Cardiovascular disease is the leading global cause of death, according to the World Health Organization (WHO), accounting for more than $31 \%$ of deaths in 2016 (17.9 million) [1].
Over $43 \%$ of these deaths were due to coronary artery disease (CAD). Non-ST-segment elevation myocardial infarction (NSTEMI) accounts for up to $70 \%$ of acute coronary syndromes caused by CAD [2]. A substantial risk factor of CAD is diabetes mellitus, prevalence of which has nearly doubled in the last 30 years (from $4.6 \%$

Corresponding author:

Tomasz Skowerski MD, Department of Cardiology, School of Health Sciences, Medical University of Silesia, Katowice, Poland, phone: +48 607234 440, e-mail: tskowerski@gmail.com

Received: 12.02.2021, accepted: 24.05.2021. 
to $9.3 \%$ of the global adult population according to the International Diabetes Federation), and the majority of patients have diabetes mellitus type 2 (T2DM) [3]. The latest data show that CAD affects up to $32 \%$ of patients with T2DM [4]. It has also been proven that T2DM worsens the in-hospital and long-term prognosis of patients with NSTEMI [2]. CAD has complex etiology, including metabolic disturbances in cardiomyocytes and endothelial dysfunction. Those are especially affected in T2DM, due to insulin resistance and deregulation of glucose and fatty acid metabolism [5].

The transcriptional factor forkhead box 01 (FOXO1) is an important regulator of cell metabolism in several tissues, including the heart and the endothelium, where it is involved in cardiomyocyte metabolism (glucose and lipid metabolic pathways) [6], cell survival [7], atherosclerosis [8], and progression of diabetic cardiomyopathy [9-11].

The role of FOXO1 in genetic predisposition to CAD is unclear. A study by Kedenko et al. linked the polymorphism of FOXO1 with carotid atherosclerosis [12]. In contrast, the study by Zhao et al. on a Han Chinese population ( $n=1287$ ) showed no association of FOXO1 and the prevalence of CAD [13]. Previous studies on animal models have shown that FOXO1 expression is increased in myocardial infarction and that FOXO1 protects the myocardium through upregulation of antioxidant genes, in consequence reducing the infarct size $[14,15]$. However, the increased expression of FOXO1 in mice with insulin resistance or diabetes led to development of diabetic cardiomyopathy [11] and deletion of the FOXO1 gene in diabetic animal models prevented heart failure [9]. FOXO1 is also proven to be involved in diabetes-induced vascular remodeling in rats [16].

Vascular remodeling is also modulated by vascular growth factors among other angiopoietins, with two major ones being angiopoietin 1 (Ang-1) and angiopoietin 2 (Ang-2). One of particular interest is angiopoie-

Table I. Inclusion and exclusion criteria

\begin{tabular}{ll} 
Inclusion criteria & Age $>18$ and $<80$ years \\
& NSTEMI with or without T2DM or T2DM with- \\
& out CAD \\
\hline Exclusion criteria & Age $>80$ years \\
& Anti-inflammatory drug usage (defined as use \\
& of these drugs for longer than a week) \\
& Heart failure with reduced ejection (HFrEF) \\
& Peripheral vascular diseases \\
& Acute or chronic kidney/liver disease (esti- \\
& mated glomerular filtration rate assessed \\
& with CKD-EPI (eGFR) $<60 \mathrm{ml} / \mathrm{min} / 1.73$ m²) \\
& AST and/or ALT elevated $>2 \times$ reference value \\
& Cancer \\
& History of myocardial and/or cerebral infarc- \\
& tion (within 6 months prior to enrollment in \\
& the study) \\
& Autoimmune diseases
\end{tabular}

tin-2 (Ang-2), exclusively expressed in endothelium cells, possessing an opposite action to Ang-1, which exhibits endothelium protective properties [17]. Conditions such as chronic hyperglycemia or chronic inflammation lead to both FOXO1 transcriptional activation (PI3K/Akt signaling) [18] and Ang-2 gene expression induction in endothelial cells, binding the Tie-2 receptor, and therefore inhibiting the angiopoietin-1 because it binds to the same Tie-2 receptor, which cause destabilization of the endothelium and increase the arteriosclerosis process [19-22].

It is unclear how the FOXO1 and ANGPT2 gene expression changes during myocardial ischemia.

This study is a continuation of our previous research [23] which proved that Ang 2 serum concentration is elevated in patients with NSTEMI both with and without T2DM as well as among T2DM patients without a history of CAD.

\section{Aim}

Our objective was to evaluate ANGPT2 and FOXO1 gene expression in patients with NSTEMI, both with and without T2DM, and compare it to patients with T2DM but without CAD and to those without T2DM and without CAD. To our knowledge, no studies have evaluated ANGPT2 and FOXO1 gene expression in human patients with NSTEMI and diabetes.

\section{Material and methods}

This was a multi-center, prospective study that included patients consecutively hospitalized in cardiology or diabetology wards between January 2017 and July 2017 due to NSTEMI (emergency admission) or T2DM. The control group consisted of patients with arterial hypertension and suspected of cardiovascular diseases which were finally unconfirmed.

Inclusion and exclusion criteria are presented in Table I. In brief, 138 patients who fulfilled inclusion/exclusion criteria were enrolled in the study; 11 patients were not included for further analysis (due to incomplete data).

There were 127 patients divided into 4 groups: Group A: 24 patients with NSTEMI and T2DM; Group B: 43 patients with NSTEMI without T2DM; Group C: 30 patients with T2DM, without a history of CAD; Group D (control group): 30 patients without CAD and without T2DM.

On the day of hospital admission patients had $10 \mathrm{ml}$ of peripheral blood drawn for the biochemical analysis with high-sensitivity C-reactive protein (hs-CRP) and troponin level (TnT), and anthropometric and demographic data, as well as past medical history, were collected. Electrocardiography and echocardiography with assessment of ejection fraction (EF\%) were performed. The blood analysis included: FOXO1 and ANGPT2 gene expression (qPCR analysis) and serum level of Ang-2 and glycated hemoglobin $\left(\mathrm{HbA}_{1 \mathrm{c}}\right)$. Patients from groups $\mathrm{C}$ and $\mathrm{D}$ had no 
history of CAD, presented no symptoms of CAD and had no ischemic changes in ECG or ECHO (preserved ejection fraction and no features of having undergone myocardial ischemia).

The study was approved by the Medical University of Silesia Ethics Committee and conformed to the Declaration of Helsinki (KNW/0022/ KB1/28/16; KNW/0022/ KB1/28/I/16/17). Informed written consent was obtained from every patient enrolled in the study.

\section{FOXO1 and ANGPT2 gene expression analysis}

The buffy coat was obtained by centrifugation of peripheral blood and stored in aliquots at $-80^{\circ} \mathrm{C}$ until further analysis.

RNA was extracted from the buffy coat using a Maxwell 16 MDX device (PROMEGA Wisconsin, USA) with Cell Lysis Solution (Kit A793A PROMEGA, Wisconsin, USA) and Lysis Buffer (Kit MC501C, PROMEGA Wisconsin, USA) according to the manufacturer's protocol [24].

RNA concentration was determined spectrophotometrically by measuring the absorbance at $260 \mathrm{~nm}$ and the purity was estimated by the ratio A260/A280 nm and A260/A230 nm (Denovix DS-11, Denovix Inc., Wilmington, Delaware, USA).

The purified RNA was reverse transcribed to cDNA using a SuperScript VILO MasterMIX kit (Kit 11755-250, Thermo Fisher Scientific, California, USA) according to the manufacturer's protocol [25].

ACTB and TBP genes were initially selected as candidates for the reference gene. Quantitative analysis of the FOXO1, ANGPT2, actin beta (ACTB) gene and TATA box binding protein (TBP) genes was carried out by two-step reverse transcription real-time quantitative $P C R$ assay (qPCR) using a LightCycler 96 Instrument (ROCHE) with a thermal profile set as follows: $95^{\circ} \mathrm{C} / 10 \mathrm{~min}$ then $55 \mathrm{cy}$ cles of $95^{\circ} \mathrm{C} / 15 \mathrm{~s}, 60^{\circ} \mathrm{C} / 60 \mathrm{~s}$.

FOXO1, ANGPT2, TBP and ACTB gene expression levels were measured using TaqMan chemistry and the primer/ probe pairs with the following assay IDs - Hs01054576 m1 (FOXO1), Hs01048042 (ANGPT2), Hs00427620 m1 (TBP), Hs99999903 (ACTB).

After validation using RefFinder [26] the TBP gene proved to be more stable and was used as a reference gene. The $2^{-\Delta \Delta C a}$ method was used to analyze FOXO1 and ANGPT2 expression levels relative to the TBP reference gene control [27].

If a PCI was performed, the same blood serum analysis was repeated at discharge to assess potential changes of FOXO-1 and ANGPT2 gene expressions after PCI.

\section{Blood serum collection and analysis}

The Ang-2 levels were measured using enzyme-linked immunosorbent assay (ELISA) (RayBio ANGPT2 ELISA Kit, RayBiotech Inc. Norcross USA, Catalog \#: ELH-Angiopoietin2). $\mathrm{HbA}_{1 c}$ value was analyzed with a high-performance liquid chromatography method. Serum high-sensitivity troponin concentration was measured using the chemiluminescence method.

\section{Angiographic complexity of CAD}

Patients from groups A and B (NSTEMI with/without T2DM) underwent urgent $(<24 \mathrm{~h})$ coronarography and the SYNTAX score grading system was used to determine the complexity of coronary artery disease and in order to qualify patients for $\mathrm{PCl}$ or CABG. Each patient with NSTEMI also had the GRACE 2.0 score calculated in order to estimate the in-hospital outcome. The SYNTAX score, GRACE 2.0 score, number of implanted stents (in the case of $\mathrm{PCl}$ ) and type of intervention ( $\mathrm{PCl}$ or $\mathrm{CABG}$ ) were further analyzed and compared with FOXO1 and ANGPT2 gene expression.

\section{Statistical analysis}

All statistical calculations were performed using Microsoft Office Excel, Statistica 12.0 (StatSoft Inc., USA) software and R software (version 3.4.4). The Shapiro-Wilk normality test was used to establish the distribution of quantitative variables. Descriptive statistics for continuous parameters of normal distribution are presented as the arithmetic means (standard deviation (SD)). Median values with interquartile range are presented for continuous data that did not have normal distribution. Absolute values and percentages are given for categorical and qualitative variables. The Kruskal-Wallis test was used to determine the significance of differences between multiple groups, and the Wilcoxon test was employed for the cases with two groups. Correlations between pairs of continuous variables were fitted using the Spearman rho coefficient. Logistic regression analyses were performed to determine whether FOXO1 or ANGPT2 gene expression levels were independently associated with the complexity of CAD. The prognostic accuracy of NSTEMI of FOXO1 gene expression was assessed using an area under the receiver operating characteristic (ROC) curve. The threshold of statistical significance for all tests was set at $p<0.05$.

\section{Results}

Baseline group characteristics and results are presented in Table II. There were 127 patients included in the final analysis (84 males - 66\%). Age of patients in groups $A, B$, and $C$ did not differ significantly between each group, though the control group was significantly younger in comparison to groups $A, B$ and $C(p<0.05)$. T2DM patients (groups $A$ and $C$ ) had higher $\mathrm{BMI}$ and $\mathrm{HbA}_{1 \mathrm{c}}$ in comparison to patients without T2DM (groups $\mathrm{B}$ and $\mathrm{D}, p<0.05)$. Among patients with NSTEMI, both groups included more active smokers than among patients without NSTEMI. The level of cholesterol was lower in patients with NSTEMI and T2DM (group A) than in patients from groups $\mathrm{B}, \mathrm{C}, \mathrm{D}, p<0.05$. 
Table II. Baseline group characteristics and results. Group A - NSTEMI with T2DM; group B - NSTEMI without T2DM; group C - T2DM; group D - control subjects

\begin{tabular}{|c|c|c|c|c|c|}
\hline Parameter & A $(n=24)$ & B $(n=43)$ & $C(n=30)$ & $\mathrm{D}(n=30)$ & $P$-value \\
\hline Age [years] & $66.5(60 ; 70)$ & $66(58 ; 72)$ & $60(53 ; 67)$ & $47(37 ; 62)$ & NS \\
\hline Male, $n(\%)$ & $20(83.3)$ & $32(74.4)$ & $16(53.3)$ & $16(53.3)$ & - \\
\hline BMI $\left[\mathrm{kg} / \mathrm{m}^{2}\right]$ & $30.3(27 ; 33)$ & $28.1(25.6 ; 31.7)$ & $32(26.7 ; 34.2)$ & $27.3(25.7 ; 30.1)$ & $<0.05$ \\
\hline Active smoker, $n(\%)$ & $16(66.7)$ & $29(67.4)$ & $9(30)$ & $9(30)$ & - \\
\hline Cholesterol [mg/dl] & $147(125.2 ; 167.5)$ & $173(146 ; 211)$ & $179(163 ; 204)$ & $165(148 ; 189)$ & $<0.05$ \\
\hline $\mathrm{HDL}[\mathrm{mg} / \mathrm{dl}]$ & $39(31.5 ; 48.2)$ & $43(37 ; 63)$ & $46(33.8 ; 52.8)$ & $50(42 ; 57)$ & $<0.05$ \\
\hline $\mathrm{LDL}[\mathrm{mg} / \mathrm{dl}]$ & $72(56.8 ; 95.5)$ & $106(71 ; 135.5)$ & $93.5(63.8 ; 118.2)$ & $95(79 ; 123)$ & $<0.05$ \\
\hline Creatinine $[\mathrm{mg} / \mathrm{dl}]$ & $0.89(0.79 ; 0.98)$ & $0.9(0.78 ; 1.01)$ & $0.8(0.7 ; 0.97)$ & $0.87(0.73 ; 0.99)$ & NS \\
\hline Diabetes mellitus, $n(\%)$ & $24(100)$ & NA & $30(100)$ & NA & - \\
\hline $\mathrm{HbA}_{\mathrm{lc}}[\mathrm{mg} / \mathrm{dll}]$ & $6.3(5.7 ; 7.1)$ & $5.4(5.3 ; 5.7)$ & $8.9(8.1 ; 9.6)$ & $5.2(5.1 ; 5.5)$ & $<0.05$ \\
\hline HOMA-IR & $3.6(2 ; 5.4)$ & $1.7(1.3 ; 2.8)$ & $5.8(1.8 ; 9.7)$ & $2.7(1.8 ; 8.9)$ & $<0.05$ \\
\hline Ejection fraction, \% & $44(37.2 ; 50)$ & $50(44 ; 55)$ & $60(55 ; 60)$ & $57(50 ; 60)$ & NS \\
\hline GRACE 2.0 score & $110.5(95 ; 118.8)$ & $110(92.5 ; 120)$ & NA & NA & NS \\
\hline SYNTAX score & $17(9.5 ; 23.6)$ & $10(1 ; 18.5)$ & NA & NA & $<0.05$ \\
\hline ANGPT2 relative expression & $1.94(1.85 ; 19.4) n=3$ & $1.41(0.1 ; 1.41) n=3$ & $1.11(0.34 ; 1.11) n=4$ & $0.62 n=1$ & NS \\
\hline FOXO1 relative expression & $1.49(0.8 ; 2.51)$ & $1.27(0.85 ; 1.85)$ & $1.11(0.8 ; 1.39)$ & $1.08(0.59 ; 1.34)$ & 0.14 \\
\hline ANG-2 [pg/ml] serum level & $1725(1042 ; 2862)$ & $1786(1074 ; 2418)$ & $1983(1152 ; 2610)$ & $822(536 ; 1172)$ & $<0.05$ \\
\hline
\end{tabular}

\section{Relative expression of ANGPT2 and FOXO1 genes}

The ANGPT2 gene expression in buffy coat in the analyzed samples was very low and detectable only in 11 patients from all groups (8.66 \% of all patients) (Table III).

The FOXO1 gene expression in buffy coat was detectable in all analyzed samples.

Kruskal-Wallis test was used to determine significance of differences between multiple groups and showed no significant difference of FOXO1 relative expression (Table III).

However, the level of FOXO1 expression was significantly higher in patients with NSTEMI (groups A and B combined, median relative expression $=1.39$ ) than in non-NSTEMI patients (groups C and D combined, median $=1.09)(W=1578, p<0.05)($ Table III).
In the between-group comparison significantly higher FOXO1 expression was observed in patients with NSTEMI and T2DM (group A) in comparison to the control group (1.49 vs. $1.08, p<0.05)$.

\section{Angiographic complexity of CAD}

We compared the complexity of coronary artery disease in patients with NSTEMI and with/without T2DM. Diabetic patients had a higher SYNTAX score (17 points vs. 10 points; $p<0.05)$ and were more often qualified for CABG $(29.2 \%$ vs. $16.3 \%$; $p<0.05)$. In both groups PCI was performed in a similar percentage of patients (54\% vs. $60 \% ; p=N S$ ). After statistical analysis (logistic regression model, Spearman correlation) no link between FOXO1 expression and the severity of CAD (SYNTAX score), GRACE score, number of implanted stents or type of intervention (PCI vs. CABG) was found.

Table III. Kruskal-Wallis test; comparison of FOXO-1 expression in study groups - median, $1^{\text {st }}$ and $3^{\text {rd }}$ quartile. Group A - NSTEMI and T2DM; group B - NSTEMI without T2DM; group C - T2DM; group D - control subjects

\begin{tabular}{|c|c|c|c|c|}
\hline \multirow{2}{*}{$\frac{\text { Group }}{A+B \text { vs. } C+D}$} & \multicolumn{3}{|c|}{ FOXO-1 expression } & \multirow{2}{*}{$\frac{P \text {-value }}{<0.05}$} \\
\hline & 1.39 & vs. & 1.09 & \\
\hline A vs. B & $1.49(0.8 ; 2.51)$ & vs. & $1.27(0.85 ; 1.85)$ & NS \\
\hline A vs. C & $1.49(0.8 ; 2.51)$ & vs. & $1.11(0.8 ; 1.39)$ & NS \\
\hline A vs. D & $1.49(0.8 ; 2.51)$ & vs. & $1.08(0.59 ; 1.34)$ & $<0.05$ \\
\hline B vs. C & $1.27(0.85 ; 1.85)$ & VS. & $1.11(0.8 ; 1.39)$ & NS \\
\hline B vs. D & $1.27(0.85 ; 1.85)$ & vs. & $1.08(0.59 ; 1.34)$ & 0.092 \\
\hline C vs. D & $1.11(0.8 ; 1.39)$ & vs. & $1.08(0.59 ; 1.34)$ & NS \\
\hline
\end{tabular}


The FOXO1 relative expression was not correlated with GRACE 2.0 score or SYNTAX score of NSTEMI patients. Also, we did not observe any significant change in FOXO1 gene expression after successful angioplasty (Figure 1).

The prognostic accuracy of FOXO1 gene expression in detecting NSTEMI determined with the area under the curve (AUC $=0.607$; Figure 2 ) was not a significant predictor of NSTEMI in the logistic regression model $(p=0.308)$.

\section{Discussion}

We observed a very low ANGPT2 relative gene expression in buffy coat (only in $8.66 \%$ of patients) - it was under the cut-off value $(C t>40)$ of the qPCR reaction. These results are in line with a previous publication by Ehrlich et al. - ANGPT2 had the lowest expression in blood relative to other tissues [28].

We found no correlation of ANGPT2 relative gene expression with Ang-2 concentration, although the Ang-2 serum level in peripheral blood was elevated in patients with NSTEMI and T2DM [23].

The next step in our study was to evaluate the FOXO1 relative gene expression, given that the $\mathrm{FOXO} 1$ protein is involved in vascular remodeling and has been previously reported to be elevated in myocardial infarction in animal models and to promote cardiac dysfunction [9, 14, 15, 2931]. The FOXO1 gene expression was under the Ct cut-off value in all study patients. The main finding of this study is that FOXO1 gene expression in blood peripheral cells is elevated in patients with NSTEMI compared to patients without NSTEMI (both with T2DM and without T2DM; Table III, relative expression -1.39 vs. 1.09; $p<0.05$ ). The percutaneous coronary intervention had no significant effect on FOXO1 gene expression and did not correlate with the angiographic complexity of CAD in patients with NSTEMI.

A recent study by Hueso et al. [32] reported downregulation of FOXO1 gene expression in patients with stable CAD. Subjects in this study ( $n=10$ with) were undergoing scheduled CABG surgery. This study combined with the results of ours can lead to the conclusion that FOXO1 gene expression is downregulated in stable CAD and upregulated in myocardial infarction.

Accumulating evidence has demonstrated that FOXO1 as a key angiogenic regulator also participates in the development and progression of diabetes. FOXO1 expression level has been found to be elevated in many tissues of diabetic mice and involved in diabetes-induced oxidative stress and cell apoptosis [7, 33]. In our study we found no significant differences between FOXO1 gene expression in patients with T2DM (group C) and the control subjects (group D) (Table III). Also, no correlation between $\mathrm{HbA}_{1 \mathrm{c}}$ and FOXO1 gene expression was found.

\section{Conclusions}

On the basis of our results we can conclude that analyzing the ANGPT2 gene expression in peripheral blood

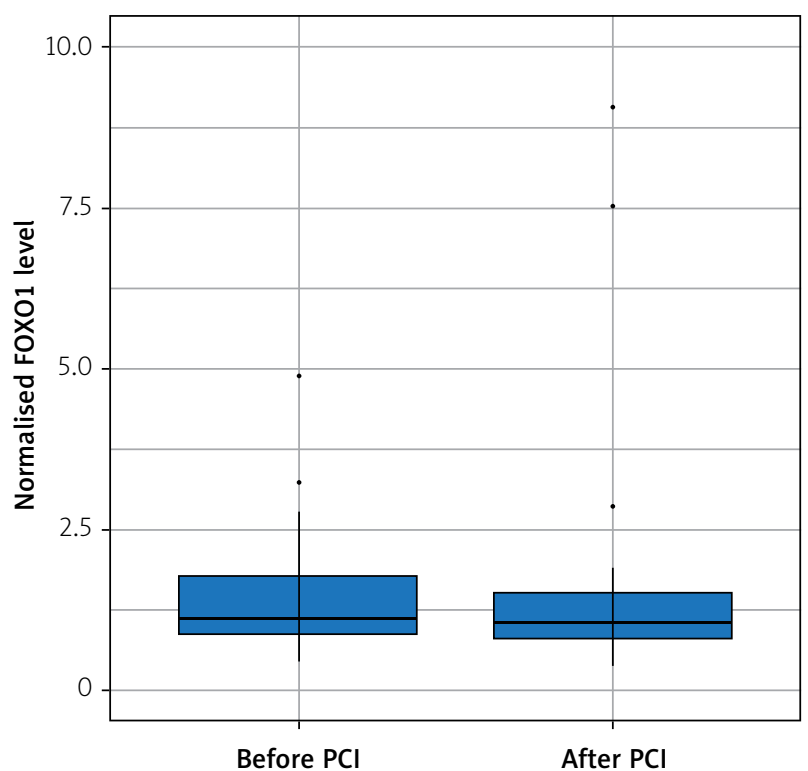

Figure 1. FOXO1 gene expression after successful angioplasty

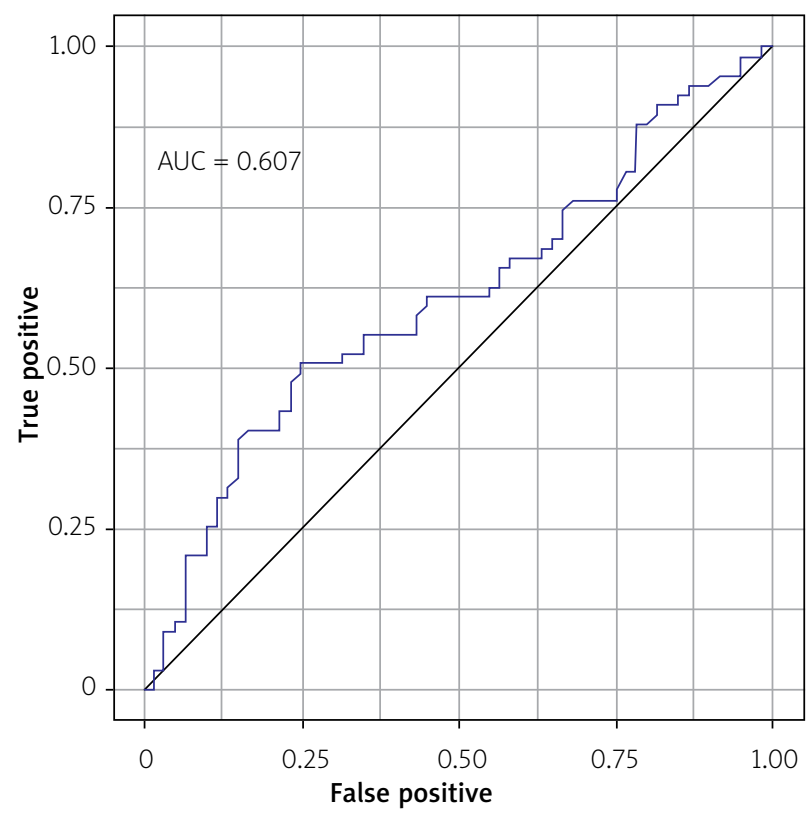

Figure 2. Prognostic accuracy of FOXO1 gene expression in detecting NSTEMI determined with area under the curve (AUC $=0.607$ )

has no role in assessment of CAD complexity among patients with and without T2DM. FOXO1 gene expression in blood peripheral cells is elevated in patients with NSTEMI regardless of the presence of T2DM. FOXO1 expression does not decrease after percutaneous coronary intervention and is not correlated with the severity of CAD in patients with NSTEMI.

\section{Acknowledgments}

This research was funded by the Medical University of Silesia. 


\section{Conflict of interest}

The authors declare no conflict of interest.

\section{References}

1. WHO, WHF, WSO. Global atlas on cardiovascular disease prevention and control. World Heal Organ 2011; 2-14.

2. Piepoli MF, Hoes AW, Agewall S, et al. 2016 European Guidelines on cardiovascular disease prevention in clinical practice. Eur Heart J 2016; 37: 2315-81.

3. Internation Diabetes Federation. IDF Diabetes Atlas, 9th edn. Brussels, Belgium Int. Diabetes Fed 2019.

4. Einarson TR, Acs A, Ludwig C, et al. Prevalence of cardiovascular disease in type 2 diabetes: a systematic literature review of scientific evidence from across the world in 2007-2017. Cardiovasc Diabetol BioMed Central 2018; 17: 83.

5. Arneth B, Arneth R, Shams M. Metabolomics of type 1 and type 2 diabetes. Int J Mol Sci 2019; 20: 2467.

6. Puthanveetil P, Wang Y, Zhang D, et al. Cardiac triglyceride accumulation following acute lipid excess occurs through activation of a FoxO1-iNOS-CD36 pathway. Free Radic Biol Med 2011; 51: 352-63.

7. Puthanveetil P, Wan A, Rodrigues B. FoxO1 is crucial for sustaining cardiomyocyte metabolism and cell survival. Cardiovasc Res 2013; 97: 393-403.

8. Tanaka J, Qiang L, Banks AS, et al. Foxo1 links hyperglycemia to LDL oxidation and endothelial nitric oxide synthase dysfunction in vascular endothelial cells. Diabetes 2009; 58: 2344-54.

9. Qi Y, Zhu Q, Zhang K, et al. Activation of foxo1 by insulin resistance promotes cardiac dysfunction and $\beta$ myosin heavy chain gene expression. Circ Hear Fail 2015; 8: 198-208.

10. Kandula V, Kosuru R, Li H, et al. Forkhead box transcription factor 1: role in the pathogenesis of diabetic cardiomyopathy. Cardiovasc Diabetol 2016; 15: 44.

11. Battiprolu PK, Hojayev B, Jiang N, et al. Metabolic stress-induced activation of FoxO1 triggers diabetic cardiomyopathy in mice. J Clin Invest 2012; 122: 1109-18.

12. Kedenko L, Lamina C, Kedenko I, et al. Genetic polymorphisms at SIRT1 and FOXO1 are associated with carotid atherosclerosis in the SAPHIR cohort. BMC Med Genet 2014; 15: 112.

13. Zhao Y, Yu Y, Tian X, et al. Association study to evaluate FoxO1 and $\mathrm{FoxO}_{3}$ gene in CHD in Han Chinese. PLoS One 2014; 9: e86252.

14. Shao D, Zhai P, Del Re DP, et al. A functional interaction between Hippo-YAP signalling and FoxO1 mediates the oxidative stress response. Nat Commun 2014; 5: 3315.

15. Lee SJ, Lee C, Kang S, et al. Angiopoietin-2 exacerbates cardiac hypoxia and inflammation after myocardial infarction. J Clin Invest 2018; 128: 5018-33.

16. Liu J. FoxO1-induced inflammation contributes to the development of diabetic vascular remodeling. FASEB J 2019; 33: 512.2.

17. Yuan HT, Khankin EV, Karumanchi SA, et al. Angiopoietin 2 is a partial agonist/antagonist of Tie2 signaling in the endothelium. Mol Cell Biol 2009; 29: 2011-22.

18. Kim M, Allen B, Korhonen EA, et al. Opposing actions of angiopoietin-2 on Tie2 signaling and FOXO1 activation. J Clin Invest 2016; 126: 3511-25.

19. Bartels S, Klein C, Felcht M, et al. Angiopoietin-2 differentially regulates angiogenesis through TIE2 and integrin signaling. J Clin Invest 2012; 122: 1991-2005.
20. Fiedler U, Reiss Y, Scharpfenecker M, et al. Angiopoietin-2 sensitizes endothelial cells to TNF- $\alpha$ and has a crucial role in the induction of inflammation. Nat Med 2006; 12: 235-9.

21. Scholz A, Plate KH, Reiss Y. Angiopoietin-2: a multifaceted cytokine that functions in both angiogenesis and inflammation. Ann N Y Acad Sci 2015; 1347: 45-51.

22. Schuldt EA, Lieb W, Dörr M, et al. Circulating angiopoietin-2 and its soluble receptor Tie-2 concentrations are related to inflammatory markers in the general population. Cytokine 2018; 105: 1-7.

23. Skowerski T, Nabrdalik K, Kwiendacz H, et al. Angiopoietin-2 as a biomarker of non-ST-segment elevation myocardial infarction in patients with or without type 2 diabetes. Arch Med Sci DOI: https://doi.org/10.5114/aoms.2019.89201.

24. Promega. Techinal Manual Wizard ${ }^{\circledR}$ Genomic DNA Purification Kit Wizard ${ }^{\circledR}$ Genomic DNA Purification Kit. Promega Corp 2017. 25. Thermofisher. SuperScript TM VILO TM MasterMix: 0-3.

26. Xie F, Xiao P, Chen D, et al. miRDeepFinder: a miRNA analysis tool for deep sequencing of plant small RNAs. Plant Mol Biol 2012; 80: 75-84.

27. Livak KJ, Schmittgen TD. Analysis of relative gene expression data using real-time quantitative PCR and the $2-\Delta \Delta C T$ method. Methods 2001; 25: 402-8.

28. Ehrlich KC, Lacey M, Ehrlich M. Tissue-specific epigenetics of atherosclerosis-related ANGPT and ANGPTL genes. Epigenomics 2019; 11: 169-86.

29. Boal F, Timotin A, Roumegoux J, et al. Apelin-13 administration protects against ischaemia/reperfusion-mediated apoptosis through the FoxO1 pathway in high-fat diet-induced obesity. Br J Pharmacol 2016; 173: 1850-63.

30. Kappel BA, Stöhr R, De Angelis L, et al. Posttranslational modulation of FoxO1 contributes to cardiac remodeling in post-ischemic heart failure. Atherosclerosis 2016; 249: 148-56.

31. López B, González A, Ravassa S, et al. Circulating biomarkers of myocardial fibrosis: the need for a reappraisal. J Am Coll Cardiol 2015; 65: 2449-56.

32. Hueso M, Mallén A, Casas Á, et al. Integrated miRNA/mRNA counter-expression analysis highlights oxidative stress-related genes CCR7 and FOXO1 as blood markers of coronary arterial disease. Int J Mol Sci 2020; 21: 1943.

33. Shi Y, Fan S, Wang D, et al. FOXO1 inhibition potentiates endothelial angiogenic functions in diabetes via suppression of ROCK1/Drp1-mediated mitochondrial fission. Biochim Biophys Acta 2018; 1864: 2481-94. 